Research Article

Adja B. R. Touré*, Elisa Mele, and Jamieson K. Christie

\title{
Atomic-scale clustering inhibits the bioactivity of fluoridated phosphate glasses
}

https://doi.org/10.1515/bglass-2019-0007

Received Jan 21, 2019; revised Aug 07, 2019; accepted Oct 02, 2019

\begin{abstract}
Here, molecular dynamics simulations have been carried out on phosphate glasses to clarify the previously debated influence of fluoride on the bioactivity of these glasses. We developed a computationally advanced inter-atomic force field including polarisation effects of the fluorine and oxygen atoms. Structural characterisations of the simulated systems showed that fluoride ions exclusively bond to the calcium modifier cations creating clusters within the glass structure and therefore decreasing the bioactivity of fluoridated phosphate glasses, making them less suitable for biomedical applications.
\end{abstract}

Keywords: Bioactive glasses, Fluorine, Phosphate, Molecular Dynamics, Force Field, Shell Model

\section{Introduction}

Bioactive phosphate glasses (PGs) are amorphous materials which induce a physiological response once implanted in the body [1, 2]. In addition, PGs completely dissolve in an aqueous environment with a dissolution behaviour controlled by the glass composition [3], hence their numerous biomedical applications [4], including neural repair [5], dental implants [6], tissue engineering [7-9], bone fracture fixation $[10,11]$ and drug delivery devices $[12,13]$.

The structure of PGs is based on the $\mathrm{PO}_{4}$ tetrahedron. Oxygen atoms can play two different roles: as a bridging

\footnotetext{
${ }^{\star}$ Corresponding Author: Adja B. R. Touré: Department of Materials, School of Aeronautical, Automotive, Chemical and Materials Engineering, Stewart Miller Building Loughborough University, Loughborough LE11 3TU United Kingdom; Email: a.toure@lboro.ac.uk Elisa Mele: Department of Materials, School of Aeronautical, Automotive, Chemical and Materials Engineering, Stewart Miller Building Loughborough University, Loughborough LE11 3TU United Kingdom; Email: E.Mele2@lboro.ac.uk

Jamieson K. Christie: Department of Materials, School of Aeronautical, Automotive, Chemical and Materials Engineering, Stewart Miller Building Loughborough University, Loughborough LE11 3TU United Kingdom; Email: J.K.Christie@lboro.ac.uk
}

oxygen (BO) when it links two tetrahedra via a P-O-P bond and as a non-bridging oxygen (NBO) if no such bond exists. The mean number of $\mathrm{BO}$ atoms per phosphorus atom is the network connectivity (NC) [14]. The NC is the main structural parameter affecting the bioactivity of the PGs. Amorphous glasses allow more flexibility in composition because they are not dependent on a specific stoichiometry as crystals are. Therefore, different amounts of ions with physiological activity and/or therapeutic properties can also be incorporated into glasses [7, 15-19].

In this paper, we focus on bioactive glasses containing fluoride which is beneficial in dentistry as it prevents caries (tooth decay) [2, 20, 21]. Fluoride induces the enhancement of tooth enamel remineralisation, the inhibition of enamel demineralisation and bacterial infections. Fluoride ions cause fluorapatite to form in physiological solutions [22, 23]. This fluoridated form of hydroxyapatite, which is the main component of the tooth, is more stable against acid attacks than hydroxyapatite [24].

Our aim in this paper is to understand how the incorporation of fluoride ions affects the bioactivity. Conventional definitions of bioactivity often refer to a material's ability to mineralise apatite on its surface either in vitro or in vivo after implantation. Although there is evidence for apatite formation on the surface of phosphate glasses [25], typical compositions dissolve too fast for this to be a particularly relevant descriptor of the glass's properties. When referring to the bioactivity of these compositions, we refer more broadly to the reactions which it undergoes when implanted in vivo. For these compositions, we are able to characterise the changes in bioactivity caused by fluoridation of the glass by connecting changes in the atomic structure of the glass to its dissolution rate.

In order to obtain the most detailed representation of its structural organisation, the structure of PGs is investigated using computer simulation. Molecular dynamics simulations have often been used to complement experimental data by highlighting different effects [26-32] which can be linked to the bioactivity such as the coordination environment of network formers, tendency of modifiers to form clusters, inhomogeneities and the existence of chain and ring nanostructures. Additionally, in

¿ Open Access. (C) 2019 A. B. R. Touré et al., published by De Gruyter. (CC) BY 4.0 License 
some cases the acquisition of experimental measurements have proved to be complicated, time consuming or even unattainable [33, 34]. Therefore, simulations are an efficient and reliable way to map relevant structural features and link the composition of PGs to their structure and solubility.

When considering the effect of fluoride incorporation, there are different phenomena which affect the bioactivity. The single charge on $\mathrm{F}^{-}$implies that a bridging fluorine atom cannot exist and hence that any F-P bonding in which the fluorine atom would take the place of an oxygen atom in a $\mathrm{PO}_{4}$ (now $\mathrm{PO}_{3} \mathrm{~F}$ ) tetrahedron will reduce the $\mathrm{NC}$ and hence increase dissolution rate. Conversely, any fluoride clustering to the modifier ions will separate the glass into modifier-rich (phosphate-poor) and modifierpoor (phosphate-rich) regions, which will not change the average NC, but will cause local changes which might affect the dissolution rate of the glass. The NC will also change depending on how exactly the fluoride is incorporated, for example, fluoridation of silicate glass is observed to reduce the rate of apatite formation [35] when $\mathrm{CaF}_{2}$ is substituted directly for $\mathrm{CaO}$, but fluoridation in this way also causes an increase in $\mathrm{NC}$ which would be expected to decrease the rate of apatite formation and hence bioactivity. When $\mathrm{CaF}_{2}$ is added to a calcium silicate glass and $\mathrm{NC}$ is kept constant, a decrease in rate of apatite formation and hence bone-bonding ability is observed [36], and in vivo experiments on a F-containing silicate glass showed the "absence of a continuous Si-rich layer" [37], one of the necessary steps in the bioactive mechanism. More recent work on silicate glass compositions with different fluoride content but constant NC has shown that high-F content glasses often crystallize fluorite at the expense of apatite [22], and other work has shown that the rate of apatite formation increases with increasing phosphate content in a silicate glass [23]. A full understanding of the connections between glass composition, structure, and bioactivity continues to be challenging to obtain.

Two previous studies on the structure of fluoridated PGs have been contradictory. Classical molecular dynamics simulations saw no F-P bonding and concluded that the addition of fluorine leads to the formation of clusters [25]. Another study used quantum-mechanical modelling and observed a significant amount of F-P bonding implying negligible clustering [29]. To clarify the influence of fluorine on the formation of clusters, we developed a classical interatomic force field with ionic charges and used a shell model to include polarisation effects [38]. The use of polarisable force fields is crucial to obtain a correct description of medium range structure and hence the bioactivity [34]. We use this force field to model accurately the atomistic structure of $\mathrm{P}_{2} \mathrm{O}_{5(50-x / 2)}-\mathrm{CaO}_{(50-x / 2)}-\mathrm{CaF}_{2(x)}$ with $x=0,2,5$ using the molecular dynamics code $[39,40]$ DL_POLY Classic [41]. The amount of $\mathrm{CaF}_{2}$ is increased while the network connectivity is kept constant to remove any effect of changing the NC on bioactivity. We show the extent of the fluorine clustering and discuss its effect on the dissolution rate, and hence bioactivity, of fluoridated PBGs.

\section{Materials and methods}

\subsection{Polarisable force field development}

In this work, we developed a force field based on the BornMayer ionic model [42] which assumes that the ions in the glass interact via long-range Coulombic internal forces, short-range two- and three-body interactions. The shortrange interactions between ions $i$ and $j$ are expressed using the Buckingham potential. The expression for the Buckingham potential energy is:

$$
U_{i j}\left(r_{i j}\right)=A_{i j} \frac{\frac{-r_{i j}}{\rho_{i j}}}{e_{i j}}-\frac{C_{i j}}{r_{i j}^{6}}
$$

where $i$ and $j$ are atomic species, $A_{i j}, \rho_{i j}$ and $C_{i j}$ are the Buckingham potential parameters of the $i-j$ interaction, and $r_{i j}$ is the distance between ions $i$ and $j$. The Coulombic energy is expressed as

$$
U_{i j}^{\text {Coulomb }}=\frac{q_{i} q_{j}}{4 \pi \epsilon_{0} r_{i j}}
$$

where $q_{i}$ is the charge of ion $i$ and $\epsilon_{0}$ is the permittivity of free space. The force field in this work takes into account

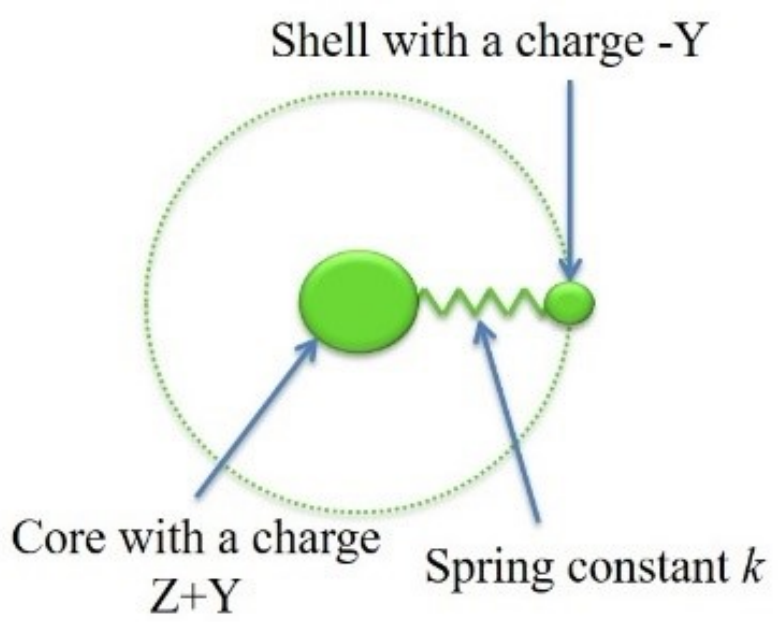

Figure 1: Schematic representation of the shell model. 
the polarisation of the oxygen and fluorine atoms using the shell model [38]. In the shell model, the polarisability is included by splitting the atom's total charge into a core of charge $(\mathrm{Z}+\mathrm{Y})$ and a shell of charge $(-\mathrm{Y})$ as shown in Figure 1.

The core and shell are linked by a harmonic spring with energy:

$$
U\left(r_{i}\right)=\frac{1}{2} k_{i} r_{i}^{2}
$$

where $k_{i}$ represents the force constant of the harmonic spring, and $r_{i}$ the distance between the core and the shell. The values for the core and shell charges for the oxygen were kept at the values derived by Catlow et al. [43] (Table 1). We used ionic charges to represent the calcium and phosphorus ions. In this model, the short-range forces are set to act on the shell whereas Coulombic forces act on both shells and cores. To sum the electrostatic interactions, we used the Ewald summation [44]. The effect of an electric field is to separate the core and the shell, giving rise to a polarisation dipole. The short-range forces affect the polarisability which becomes environment-dependent. The adiabatic shell model is used in this work, where the shell is given a mass $m$ (typically $\sim 0.2 \mathrm{amu}$ ) chosen so that the frequency of vibration of the harmonic spring is well above the frequency of vibration of the atoms in the bulk system. This is necessary to avoid significant exchange of kinetic energy between the core-shell unit and the rest of the system. Therefore, the core-shell units should have a negligible energy in comparison to the potential energy of the interatomic bonds. There can be a slow leakage of kinetic energy into the core-shell units, but this represents a negligible amount of the total kinetic energy and this can be corrected by a frictional term.

Table 1: Ion charges used in this work.

\begin{tabular}{cccc}
\hline Ion & Core (e) & Shell (e) & $\begin{array}{c}\text { Core-shell } \\
\text { interaction }\left(\mathrm{eV} \mathrm{\AA}^{-2}\right)\end{array}$ \\
\hline $\mathrm{Ca}$ & +2.000 & & \\
$\mathrm{P}$ & +5.000 & & \\
$\mathrm{O}[43]$ & +0.8482 & -2.8482 & 74.92 \\
$\mathrm{~F}[43]$ & +1.380 & -2.380 & 101.200 \\
\hline
\end{tabular}

Finally, the three-body interactions are included through a harmonic potential. This three-body potential, also known as the bond-bending term, introduces an energy penalty for any deviation from the equilibrium bond angle $\theta_{0}$ and is written as:

$$
V=\frac{1}{2} k_{i j k}\left(\theta_{i j k}-\theta_{0}\right)^{2}
$$

where $k_{i j k}$ and $\theta_{i j k}$ respectively represent the strength of the bond-bending term, and the bond angle between the three ions $i, j$ and $k$.

For transferability of the force field, already existing potentials developed for phosphate glasses [31, 45, 46] and fluorapatite $[43,47]$ were used to derive the potential. The $\mathrm{P}-\mathrm{F}_{s}, \mathrm{P}-\mathrm{F}_{s}-\mathrm{P}, \mathrm{F}_{s}-\mathrm{P}-\mathrm{F}_{s}$ and $\mathrm{F}_{s}-\mathrm{P}-\mathrm{O}_{s}$ interactions (where e.g., $\mathrm{F}_{S}$ is the shell on the fluorine atom) were fitted empirically to the structures and elastic constants of relevant crystals $\left(\mathrm{Ca}_{5} \mathrm{FO}_{12} \mathrm{P}_{3}, \mathrm{PF}_{5}, \mathrm{POF}_{3}\right.$ and $\left.\mathrm{CaF}_{2}\right)$. The energy minimisation code used is the General Utility Lattice Program (GULP) [48]. The accuracy of the potential fitting is determined by the percentage of errors between the optimised lattice structure and the experimental data, summed over all lattice parameters, corresponding to minimising $F$ in the equation below:

$$
F=\sum w_{i}\left(f_{i, c a l c}-f_{i, o b s}\right)^{2}
$$

where $w_{i}$ is the weight attributed to each parameter $f_{i}$. In this work, $w_{i}$ was equal for all parameters.

\subsection{Molecular dynamics simulations}

Once the potential was derived, classical molecular dynamics simulations were carried out using the DL_POLY code [41] to obtain an accurate atomistic description of the system. Three different compositions (Table 2) in the $\left(\mathrm{P}_{2} \mathrm{O}_{5}\right)_{(50-x / 2)}(\mathrm{CaO})_{(50-x / 2)}\left(\mathrm{CaF}_{2}\right)_{x}$ system were modelled: one with $0 \mathrm{~mol} \%$ of $\mathrm{CaF}_{2}$ (referred to as Fo), one with 2 $\mathrm{mol} \%$ of $\mathrm{CaF}_{2}$ (F2) and one with $5 \mathrm{~mol} \%$ of $\mathrm{CaF}_{2}$ (F5). The amount of $\mathrm{CaF}_{2}$ was increased while the ratio of the number of oxygen atoms to the number of phosphorus atoms, and hence the network connectivity, were kept constant. The densities of the compositions of fluoridated phosphate glasses modelled (F2 and F5 in Table 2) were not available in the literature or glass properties databases or experimentally achievable. We have therefore estimated the densities from density data on calcium phosphate bioglasses [29, 49-52], based on the fractional increase in density observed when fluorine was added to ternary silicate glasses [51], as in ref. [29].

The starting configurations consisted of quasirandomly placed atoms in a cubic box positioned so that two atoms would not be found within $80-90 \%$ of their expected interatomic separation. The molecular dynamics runs were then performed in a constant volume and temperature canonical (NVT) ensemble. The different simulations were run using a well-established and reliable methodology to model glasses [3, 25, 26, 31, 45, 53]. For each composition the initial configuration was run at 
Table 2: Simulated compositions with the corresponding densities and cell sizes.

\begin{tabular}{ccccccc}
\hline & $\mathbf{P}_{\mathbf{2}} \mathbf{O}_{\mathbf{5}} \mathbf{~ m o l} \%$ & $\mathbf{C a O} \mathbf{~ m o l} \%$ & $\mathrm{CaF}_{\mathbf{2}} \mathbf{~ m o l} \%$ & Density $\left(\mathbf{g} \cdot \mathbf{c m}^{-\mathbf{3}}\right)$ & Number of atoms & Cell size $(\AA)$ \\
\hline F0 & 50.0 & 50.0 & 0.0 & 2.589 & 2000 & 30.96 \\
F2 & 49.0 & 49.0 & 2.0 & 2.592 & 1800 & 29.90 \\
F5 & 47.5 & 47.5 & 5.0 & 2.597 & 1700 & 29.34 \\
\hline
\end{tabular}

Table 3: Two-body Buckingham potential terms.

\begin{tabular}{cccc}
\hline Interactions & $\mathbf{A}_{\mathbf{i j}}(\mathbf{e V})$ & $\boldsymbol{\rho}_{\mathbf{i j}}(\AA)$ & $\mathbf{C}_{\mathbf{i j}}\left(\mathbf{e V} \cdot \AA^{-6}\right)$ \\
\hline $\mathrm{P}-\mathrm{F}_{s}$ & 1089.25 & 0.2568 & 7.28 \\
$\mathrm{O}_{s}-\mathrm{F}_{s}[43]$ & 583833.7 & 0.2116 & 7.68 \\
$\mathrm{~F}_{s}-\mathrm{F}_{s}[43]$ & 99731834.0 & 0.1201 & 17.02 \\
$\mathrm{Ca}-\mathrm{F}_{s}[43]$ & 1272.8 & 0.29971 & 0.0 \\
$\mathrm{P}-\mathrm{O}_{s}[31]$ & 1020.0 & 0.34322 & 0.03 \\
$\mathrm{O}_{s}-\mathrm{O}_{s}[31]$ & 22764.3 & 0.149 & 27.88 \\
$\mathrm{Ca}-\mathrm{O}_{s}[31]$ & 2152.3566 & 0.309227 & 0.09944 \\
\hline
\end{tabular}

$2500 \mathrm{~K}$ until the system reaches an equilibrium. Following that, the system was gradually cooled down from $2500 \mathrm{~K}$ to $300 \mathrm{~K}$ ensuring that the system reached thermal equilibrium for each of the temperatures (2500K, 2000K, 1500K, $1000 \mathrm{~K}, 650 \mathrm{~K}$ and $300 \mathrm{~K})$. Each step was run for 40ps which corresponds to an overall cooling rate of $9-10 \mathrm{~K} / \mathrm{ps}$. This cooling rate has been shown to give reliable results in terms of medium-range structure [26]. In this paper all data are taken from averages of five independent simulations for each composition.

\section{Results and discussion}

\subsection{Polarisable force field}

The optimised potential is given in Tables 3 and 4 and the lattice parameters of the corresponding crystal structures optimised with the potential are presented in Table 5. The results obtained on the optimised structures of $\mathrm{Ca}_{5} \mathrm{FO}_{12} \mathrm{P}_{3}$, $\mathrm{POF}_{3}$ and $\mathrm{CaF}_{2}$ were in good agreement with the experimental lattice parameters, with an average error of 2.67 , 1.28 and $0.51 \%$ respectively. $\mathrm{PF}_{5}$ showed a higher percentage of error between the optimised structure and the experimental data. However, when we take into account the small amount of fluorine that we have put in our models (with a maximum of $5 \mathrm{~mol} \%$ ) a configuration where five fluorine atoms are bonded to one phosphorus atom is very unlikely.
Table 4: Three-body harmonic potential terms

\begin{tabular}{ccc}
\hline Interactions & $\mathbf{k}\left(\mathbf{e V} \cdot \mathbf{r a d}^{-\mathbf{2}}\right)$ & $\boldsymbol{\theta}(\mathbf{d e g})$ \\
\hline $\mathrm{O}_{s}-\mathrm{P}-\mathrm{O}_{s}[31]$ & 3.3588 & 109.47 \\
$\mathrm{P}-\mathrm{O}_{s}-\mathrm{P}[31]$ & 7.6346 & 141.17933 \\
$\mathrm{P}-\mathrm{F}_{s}-\mathrm{P}$ & 7.6346 & 141.17933 \\
$\mathrm{~F}_{s}-\mathrm{P}-\mathrm{O}_{s}$ & 27.853756 & 109.47 \\
$\mathrm{~F}_{s}-\mathrm{P}-\mathrm{F}_{s}$ & 0.65214 & 109.47 \\
\hline
\end{tabular}

Table 5: Percentage difference in lattice parameters between the optimised structure and experimental data

\begin{tabular}{ccccc}
\hline Crystal & $\mathbf{a}(\%)$ & $\mathbf{b}(\%)$ & $\mathbf{c}(\%)$ & Average \\
\hline $\mathrm{POF}_{3}$ & 1.90 & 1.90 & 0.50 & 1.28 \\
$\mathrm{CaF}_{2}$ & -0.51 & -0.51 & -0.51 & -0.51 \\
$\mathrm{Ca}_{5} \mathrm{FO}_{12} \mathrm{P}_{3}$ & 3.94 & 3.94 & 0.13 & 2.67 \\
$\mathrm{PF}_{5}$ & -15.59 & -15.59 & 5.95 & 12.38 \\
\hline
\end{tabular}

\subsection{Analysis of the structure of F-PGs}

Through this work, we aim to characterise the atomic structure of fluoridated phosphate glasses and the effect that the addition of fluorine will have on the bioactivity. Many studies have been carried out to study the atomistic structure of fluorine-free phosphate glasses using both experiment $[4,13,54-58]$ and simulation [3, 31]. More recent research conducted on fluoridated phosphate glasses [25,29] has found different amounts of fluorine clustering, which will affect the bioactivity. To resolve the contradictory results in those studies, we will therefore concentrate on the structure surrounding the fluorine atoms and present a full analysis of the structure of F-PG, including the extent of any fluorine clustering.

For all the compositions simulated, the partial paircorrelation function $g_{P-O}(r)$ shown in Figure 2 indicated the presence of two kinds of phosphorus bonding with bond lengths around $1.47 \AA$ and $1.61 \AA$ which respectively correspond to the double and single bond between the phosphorus and the oxygen atoms. The pair-correlation functions (Figure 2 (top)) overlap for the F0, F2 and F5 compositions, which implies that the $\mathrm{P}-\mathrm{O}$ bonding does not depend on composition, in agreement with previous simulations of fluorine-free phosphate glasses [30]. Neutron 

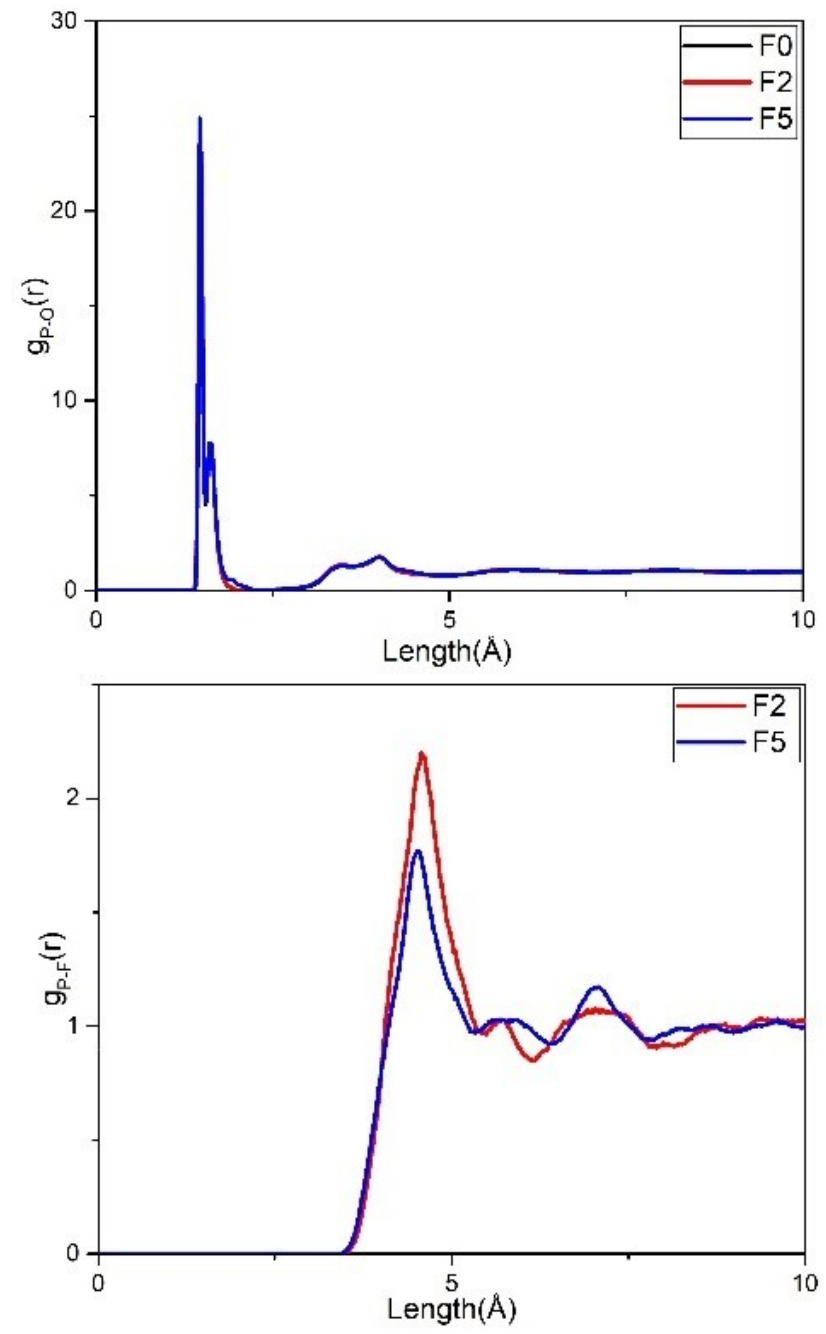

Figure 2: Partial pair-correlation functions $g_{P-O}(r)$ (top) and $g_{P-F}(r)$ (bottom) for the F0 (black), F2 (red) and F5 (blue) compositions

diffraction experiments [58] showed that the P-O distance depends on the ratio $y=n\left(M_{2 / \mu}\right) / n\left(P_{2} O_{5}\right)$ where $n(x)$ is defined by the molar amount of the species $x$ and $\mu$ represents the charge of the modifier $M$. For $y=1.04$ (our composition) the corresponding $\mathrm{P}-\mathrm{NBO}$ and $\mathrm{P}-\mathrm{BO}$ bond lengths should be around $1.51 \AA$ and $1.62 \AA$ respectively, with which we are in good agreement.

In the F2 and F5 compositions, the partial paircorrelation functions $g_{P-F}(r)$ (Figure 2) shows its first peak

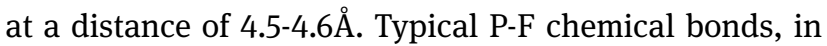
crystals such as $\mathrm{PF}_{5}$, are usually around $1.5 \AA$. We therefore observe no fluorine atoms bonding to the phosphorus atoms. The only atoms coordinated to the phosphorus are the oxygen atoms. $99.6 \%$ of the phosphorus atoms are coordinated to four oxygen atoms for all the different compositions. (The remaining $0.4 \%$ are coordinated to three or five oxygen atoms.)
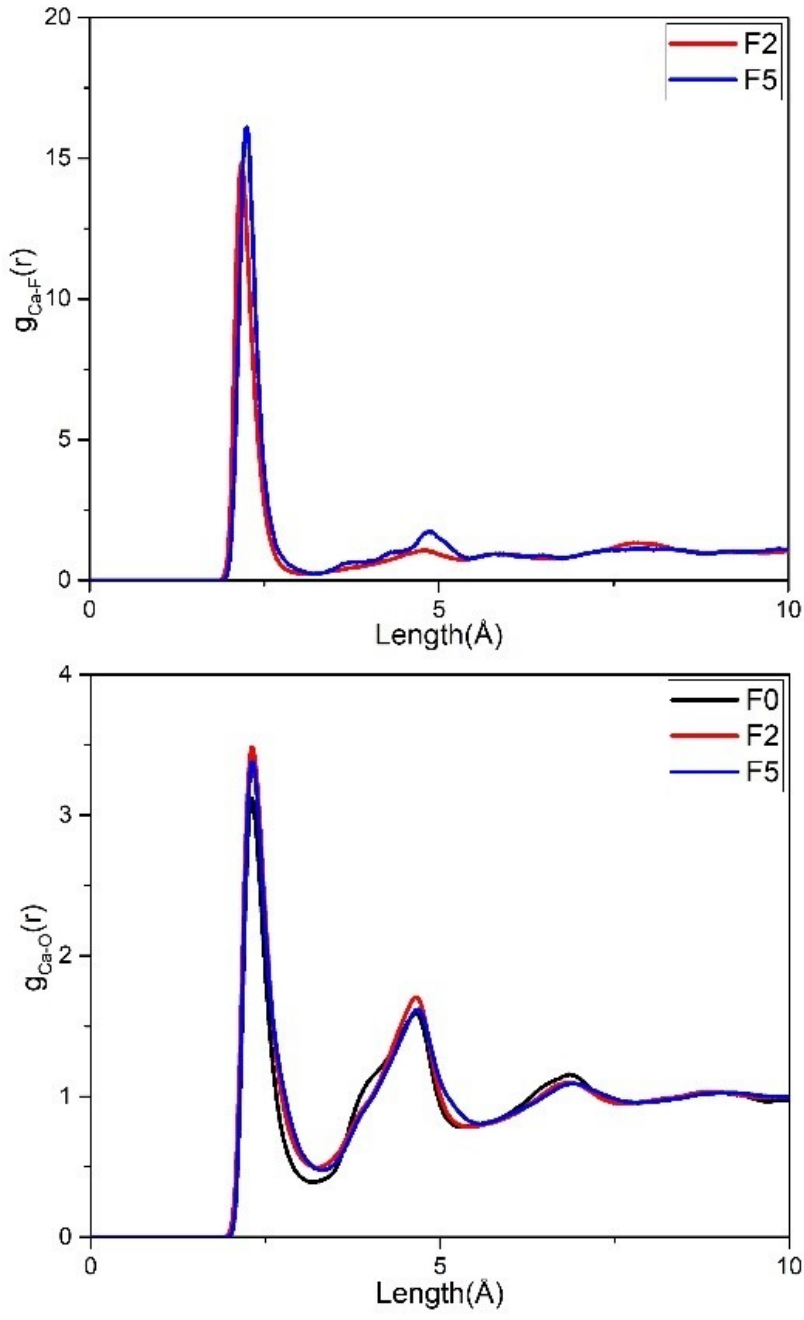

Figure 3: Partial pair-correlation functions $g_{\mathrm{Ca}-\mathrm{O}}(r)$ (top) and $g_{C a-F}(r)$ (bottom) for the F0 (black), F2 (red) and F5 (blue) compositions.

Analysis of the partial pair correlation functions $g_{\mathrm{Ca}-\mathrm{O}}(r)$ and $\mathrm{g}_{\mathrm{Ca}-\mathrm{F}}(r)$ (Figure 3) shows that the $\mathrm{Ca}-\mathrm{O}$ and Ca-F bond lengths are 2.31A and 2.21 respectively. Those values represent characteristic $\mathrm{Ca}-\mathrm{O}$ and $\mathrm{Ca}-\mathrm{F}$ distances for F-PGs [29]. The calcium atom therefore binds chemically to both fluorine and oxygen atoms. The Ca-F coordination numbers are respectively 0.19 and 0.56 for the F2 and F5 compositions and the Ca-O CNs are $6.763 \pm 0.002,6.57 \pm$ $0.01,6.05 \pm 0.01$ respectively for F0, F2 and F5. The Ca-O coordination number therefore decreases with increasing $\mathrm{CaF}_{2}$ content while the coordination number of $\mathrm{Ca}-\mathrm{F}$ increases. The fluorine atom replaces the oxygen atom in the calcium atom's first coordination shell, whereas the phosphate part of the glass structure is affected very little. 
Table 6: F-F, Ca-F, Ca-Ca and P-F clustering ratios.

\begin{tabular}{ccccc}
\hline & F2 2500K & F2 300K & F5 2500K & F5 300K \\
\hline F-F & $7.22 \pm 0.09$ & $10.64 \pm 0.24$ & $3.42 \pm 0.02$ & $6.98 \pm 0.23$ \\
Ca-F & $1.06 \pm 0.01$ & $1.12 \pm 0.01$ & $0.99 \pm 0.01$ & $1.28 \pm 0.02$ \\
Ca-Ca & $1.37 \pm 0.01$ & $1.201 \pm 0.002$ & $1.401 \pm 0.002$ & $1.209 \pm 0.002$ \\
P-F & $0.97 \pm 0.01$ & $0.874 \pm 0.005$ & $0.99 \pm 0.01$ & $0.69 \pm 0.01$ \\
\hline
\end{tabular}

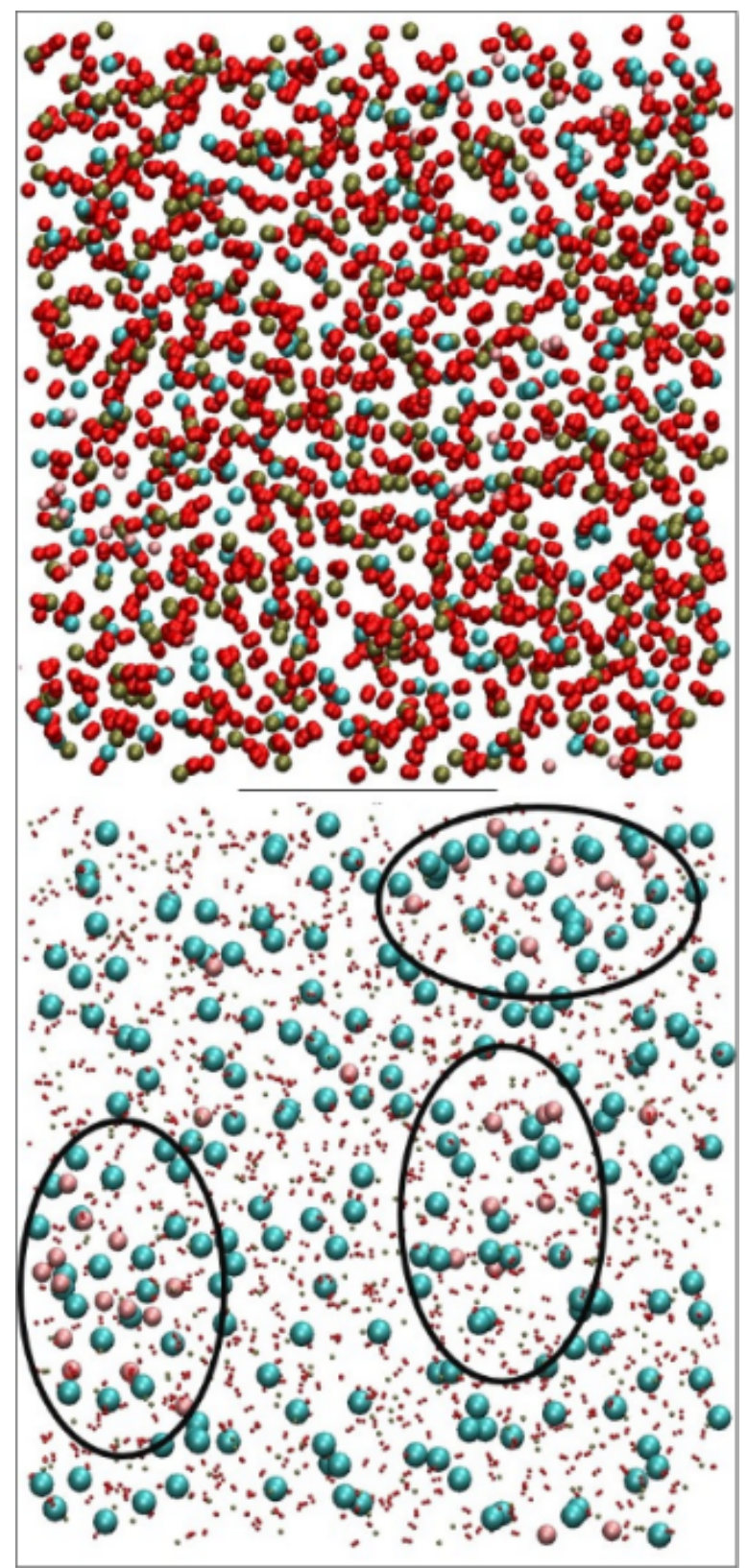

Figure 4: View of a representative F5 composition (top) with shrunk oxygen and phosphorus atoms and clusters highlighted (bottom) at $300 \mathrm{~K}$. The colours are: phosphorus (green), oxygen (red), calcium (blue) and fluorine (pink).

\subsection{Clustering}

Visualisation of the F5 composition shows clustering formed of fluorine and calcium atoms (Figure 4). These clusters show the segregation of the glass network into modifier-rich and network-rich regions, with different solubilities in a physiological environment. These clusters, which are also observed in silicate glasses [59], could lead to the formation of a discontinuous apatite layer on the surface of the bioactive glasses once implanted in the body. This uneven apatite layer could result in a decrease of the bioactivity of the glasses.

The clustering ratio of the different models can be defined as the ratio $R_{X-Y}$ of the observed total number of atoms of species $Y$ found within a coordination sphere of an atom of species $X\left(N_{X-Y, M D}\right)$ compared to that expected if the atoms were homogeneously dispersed $N_{X-Y, h o m}$. It can be calculated using the following formulas [27, 60-62].

$$
R_{X-Y}=\frac{N_{X-Y, M D}}{N_{X-Y, h o m}}=\frac{C N_{X-Y}+\delta_{(X-Y)}}{\frac{4}{3} \pi r_{C}^{3} \frac{N_{X}}{V_{b o x}}}
$$

where $\delta_{(X-Y)}$ is 1 if $X=Y$ and 0 otherwise and $r_{c}$ is a cutoff distance at which the $X-Y$ coordination number of interest $\mathrm{CN}_{X-Y}$ is calculated. $N_{X}$ represents the total number of $X$ atoms contained in the whole simulation box of volume $V_{b o x}$. The clustering ratio for the fluoridated phosphate glasses are calculated with $r_{c}=4 \AA$ and are shown in Table 6. For each composition, clustering ratios of $\mathrm{Ca}, \mathrm{F}, \mathrm{P}$, and $\mathrm{O}$ were calculated at the beginning $(2500 \mathrm{~K})$ and at the end (300K) of the simulation. We observe clear evidence of substantial fluorine clustering throughout the simulation (Figure 4). During the simulation, there is an increase of the clustering ratio for the F-F and Ca-F interactions and a small decrease for the P-F interactions. These results are in agreement with the pair-correlation functions and visualisation. They also show that the high level of fluorine clustering observed at the end of the simulation is a result of the quench: that fluorine atoms form clusters during the cooling. Previous $a b$ initio simulations [29] concluded different results in terms of phosphorus to fluorine bonding. We believe that the observed phosphorus to fluorine bonding in that study is probably due to the small size of the 
model (197 to 363 atoms) which might not be large enough to observe all kind of possible interactions, and the larger cooling rate necessary for $a b$ initio simulations.

\section{Conclusion}

This study presents classical molecular dynamics simulations of fluorine-containing phosphate glasses in the $\mathrm{P}_{2} \mathrm{O}_{5}$ $\mathrm{CaO}-\mathrm{CaF}_{2}$ system. A novel empirical force field was developed with ionic charges and the use of a shell model for polarisation effects. The classical MD simulation results showed that the addition of fluorine leads to the repolymerisation of the network into glass network-modifierrich and network-former-rich regions, with differing solubilities, as has also been reported for fluoride-containing silicate glasses [2]. This segregation is driven at the atomic scale by fluorine's preference to bond exclusively to the calcium atoms; indeed, no phosphorous to fluorine bonding was observed. We conclude that the addition of fluorine into phosphate glasses will lead to a decrease of the bioactivity of the glass. Our results imply that the segregation of the network will cause the formation of an uneven fluoroapatite layer when the glasses are implanted in the body as the ionic-rich and network-rich regions will dissolve at different rates. These variations in dissolution rate could detrimentally affect the fluoridated phosphate glasses' suitability for biomedical applications.

Acknowledgement: This paper recognises the use of the 'Hydra' High Performance System at Loughborough University and the Loughborough University School of AACME for computer time. We thank Gavin Mountjoy, Laura Swansbury and Steven Kenny for useful discussions.

Conflict of Interests: Authors state no conflict of interest.

Ethical approval: The conducted research is not related to either human or animals use.

\section{References}

[1] Knowles J.C., Phosphate based glasses for biomedical applications, J. Mater. Chem., 2003, 13, 2395.

[2] Brauer D.S., Bioactive Glasses - Structure and Properties, Angewandte Chemie Int. Ed., 2015, 54, 4160-4181.

[3] Christie J.K., Ainsworth R.I., Di Tommaso D., de Leeuw N.H., Nanoscale Chains Control the Solubility of Phosphate Glasses for Biomedical Applications, J. Phys. Chem. B, 2013, 117, 1065210657.
[4] Abou Neel E. A., Pickup D.M., Valappil S.P., Newport R.J., Knowles J.C., Bioactive functional materials: a perspective on phosphatebased glasses, J. Mater. Chem., 2009, 19, 690-701.

[5] Gilchrist T., Glasby M.A., Healy D.M., Kelly G., Lenihan D.V., McDowall K.L., Miller I.A., Myles L.M., In vitro nerve repair - in vivo. The reconstruction of peripheral nerves by entubulation with biodegradeable glass tubes - a preliminary report, Brit. J. Plast. Surg., 1998, 51, 231-237.

[6] Hench L.L., Chronology of Bioactive Glass Development and Clinical Applications, New J. Glass Ceramics, 2013, 03, 67-73.

[7] Lakhkar N.J., Lee I.H., Kim H.W., Salih V., Wall I.B., Knowles J.C., Bone formation controlled by biologically relevant inorganic ions: Role and controlled delivery from phosphate-based glasses, Adv. Drug Deliv. Rev., 2013, 65, 405-420.

[8] Ahmed I., Lewis M., Olsen I., Knowles J.C., Phosphate glasses for tissue engineering: Part 1. Processing and characterisation of a ternary-based P2O5-CaO-Na2O glass system, Biomaterials, 2004, 25, 491-499

[9] Boccaccini A.R., Erol M., Stark W.J., Mohn D., Hong Z., Mano J.F., Polymer/bioactive glass nanocomposites for biomedical applications: A review, Compos. Sci. Technol., 2010, 70, 1764-1776.

[10] Uo M., Mizuno M., Kuboki Y., Makishima A., Watari F., Properties and cytotoxicity of water soluble Na2O-CaOP2O5 glasses, Biomaterials, 1998, 19, 2277-2284.

[11] Gentleman E., Stevens M.M., Hill R.G., Brauer D.S., Surface properties and ion release from fluoridecontaining bioactive glasses promote osteoblast differentiation and mineralization in vitro, Acta Biomater., 2013, 9, 5771-5779.

[12] Pickup, D. M., Newport, R. J., Knowles, J. C., Solgel phosphatebased glass for drug delivery applications, J. Biomater. Appl., 2012, 26, 613-622.

[13] Valappil S.P., Pickup D.M., Carroll D.L., Hope C.K., Pratten J., Newport R.J., Smith M.E., Wilson M., Knowles J.C., Effect of silver content on the structure and antibacterial activity of silver-doped phosphate-based glasses, Antimicrob. Agents and Ch., 2007, 51, 4453-4461.

[14] Hill R., An alternative view of the degradation of bioglass, J. Mater. Sci. Lett., 1996, 15, 1122-1125.

[15] Maeno S., Niki Y., Matsumoto H., Morioka H., Yatabe T., Funayama A., Toyama Y., Taguchi T., Tanaka J., The effect of calcium ion concentration on osteoblast viability, proliferation and differentiation in monolayer and 3D culture, Biomaterials, 2005, 26, 4847-4855.

[16] Gentleman E., Fredholm Y.C., Jell G., Lotfibakhshaiesh N., O'Donnell M.D., Hill R.G., Stevens M.M., The effects of strontiumsubstituted bioactive glasses on osteoblasts and osteoclasts in vitro, Biomaterials, 2010, 31, 3949-3956.

[17] Shrivastava S., Bera T., Roy A., Singh G., Ramachandrarao, P., Dash, D., Characterization of enhanced antibacterial effects of novel silver nanoparticles, Nanotechnology, 2007, 18, 1-9.

[18] Yang X., Yang W., Wang Q., Li H., Wang K., Yang L., Liu W., Atomic force microscopy investigation of the characteristic effects of silver ions on Escherichia coli and Staphylococcus epidermidis, Talanta, 2010, 81, 1508-1512.

[19] Yamaguchi M., Role of zinc in bone formation and bone resorption, J. Trace Elem. Exp. Med., 1998, 11, 119-135.

[20] Featherstone J.D., The Science and Practice of Caries Prevention, J. Am. Dent. Assoc., 2000, 131, 887-899.

[21] Hoppe A., Gueldal N.S., Boccaccini A.R., A review of the biological response to ionic dissolution products from bioactive glasses 
and glass-ceramics, Biomaterials, 2011, 32, 2757-2774.

[22] Brauer D.S., Karpukhina N., O’Donnell M.D., Law R.V., Hill R.G., Fluoride-containing bioactive glasses: Effect of glass design and structure on degradation, $\mathrm{pH}$ and apatite formation in simulated body fluid, Acta Biomater., 2010, 6, 3275-3282.

[23] Mneimne M., Hill R.G., Bushby A.J., Brauer D.S., High phosphate content significantly increases apatite formation of fluoridecontaining bioactive glasses, Acta Biomater., 2011, 8, 1827-1834.

[24] Hicks J., Garcia-Godoy F., Flaitz C., Biological factors in dental caries enamel structure and the caries process in the dynamic process of demineralization and remineralization (part 2), J. Clin. Pediatr. Dent., 2005, 28, 119-124.

[25] Shaharyar Y., Wein E., Kim J.-J., Youngman R.E., Munoz F., Kim H.-W., Tilocca A., Goel A., Structure-solubility relationships in fluoride-containing phosphate based bioactive glasses, J. Mater. Chem. B, 2015, 3, 9360- 9373.

[26] Tilocca A., Cooling rate and size effects on the medium-range structure of multicomponent oxide glasses simulated by molecular dynamics, J. Chem. Phys., 2013, 139, 11450.

[27] Tilocca A., Cormack A.N., De Leeuw N.H., The Structure of Bioactive Silicate Glasses: New Insight from Molecular Dynamics Simulations, Chem. Mater., 2007, 19, 95-103.

[28] Bauchy M., Structural, vibrational, and thermal properties of densified silicates: Insights from molecular dynamics, J. Chem. Phys., 2012, 137, 15733.

[29] Christie J.K., Ainsworth R.I., De Leeuw N.H., Ab initio molecular dynamics simulations of structural changes associated with the incorporation of fluorine in bioactive phosphate glasses, Biomaterials, 2014, 35, 6164-6171.

[30] Tilocca A., Cormack A.N., Surface signatures of bioactivity: MD simulations of $45 \mathrm{~S}$ and $65 \mathrm{~S}$ silicate glasses, Langmuir, 2010, 26, 545-551.

[31] Ainsworth R.I., Di Tommaso D., Christie J.K., De Leeuw N.H., Polarizable force field development and molecular dynamics study of phosphate-based glasses, J. Chem. Phys., 2012, 137, 234502.

[32] Ruiz Hernandez S.E., Ainsworth R.I., De Leeuw N.H., Molecular dynamics simulations of bio-active phosphatebased glass surfaces, J. Non-Cryst. Solids, 2016, 451, 131- 137.

[33] Tilocca A., Structure and dynamics of bioactive phosphosilicate glasses and melts from ab initio molecular dynamics simulations, Phys. Rev. B - Condens. Matt. Mater. Phys., 2007, 76, 224202.

[34] A Tilocca A., Short- and medium-range structure of multicomponent bioactive glasses and melts: An assessment of the performances of shell-model and rigid-ion potentials, J. Chem. Phys., 2008, 129, 1-9..

[35] Lusvardi G., Malavasi G., Menabue L., Aina V., Morterra C., Fluoride-containing bioactive glasses: surface reactivity in simulated body fluids solutions, Acta Biomater., 2009, 5, 3548-62.

[36] Ebisawa Y., Kokubo T., Ohura K., Yamamuro T., Bioactivity of CaO-SiO2-based glasses:in vitro evaluation, J. Mater. Sci.: Mater. Med., 1990, 1, 239-244,.

[37] Zaffe D. , Krajewski A., Ravaglioli A., Contoli S., Study on shortterm implants of a fluorinated glass in bone, J. Mater. Sci.: Mater. Med., 1993, 4, 169-174.

[38] Dick B.G., Overhauser A.W., Theory of the Dielectric Constants of Alkali Halide Crystals, Phys. Rev., 1958, 112, 90-103.

[39] Frenkel D., Smit B., Molecular Dynamics Simulations, 2002, Elsevier.

[40] Allen M., Tildesley D., Computer Simulation of Liquids, 1988, 38, Oxford, Clarendon Press.
[41] Smith W., Forester T.R., DL-POLY-2.0: A generalpurpose parallel molecular dynamics simulation package, J. Mol. Graphics, 1996, 14, 136-141.

[42] Born M., Bormann E., Zur Gittertheorie der Zinkblende, Annalen der Physik, 1920, 367, 218-246.

[43] Catlow C.R.A., Norgett M.J., Ross T.A., Ion transport and interatomic potentials in the alkaline-earth-fluoride crystals, J. Phys. C: Solid State, 1977, 10, 1627- 1640.

[44] Ewald P.P., Die Berechnung optischer und elektrostatischer Gitterpotentiale, Annalen der Physik, 1921, 369, 253-287.

[45] Tilocca A., De Leeuw N.H., Cormack A.N., Shell-model molecular dynamics calculations of modified silicate glasses, Phys. Rev. B, 2006, 73, 104209.

[46] Sanders M.J., Leslie M., Catlow C.R.A., Interatomic potentials for SiO2, J. Chem. Soc., Chem. Commun., 1984, 4, 1271.

[47] Mkhonto D., Dde Leeuw N.H., A computer modelling study of the effect of water on the surface structure and morphology of fluorapatite: introducing a Ca10(P04)6F2 potential model, J. Mater. Chem., 2002, 12, 2633-2642

[48] Gale J.D., GULP: A computer program for the symmetry-adapted simulation of solids, J. Chem. Soc., Faraday Transactions, 1997, 93, 629-637.

[49] Pickup D.M., Guerry P., Moss R.M., Knowles J.C., Smith M.E., Newport R.J., New sol-gel synthesis of a (CaO)0.3(Na2O)0.2(P2O5)0.5 bioresorbable glass and its structural characterisation, J. Mater. Chem., 2007, 17, 4777.

[50] Brauer D.S., Anjum M.N., Mneimne M., Wilson R.M., Doweidar H., Hill R.G., Fluoride-containing bioactive glass-ceramics, J. NonCryst. Solids, 2012, 358, 1438-1442.

[51] Hill R.G., Brauer D.S., Predicting the bioactivity of glasses using the network connectivity or split network models, J. Non-Cryst. Solids, 2011, 357, 3884-3887.

[52] Venkateswara Rao G.S., Hashikala H.D., Structural, optical and mechanical properties of ternary CaO-CaF2- P2O5 glasses, J. Adv. Ceram., 2014, 3, 109-116.

[53] Christie J.K., Ainsworth R.I., Hernandez S.E.R., De Leeuw N.H., Structures and properties of phosphatebased bioactive glasses from computer simulation: a review, J. Mater. Chem. B, 2017, 5, 5297-5306.

[54] Brow R.K., Review: The structure of simple phosphate glasses, J. Non-Cryst. Solids, 2000, 263, 1-28.

[55] Brow R., Kirkpatrick R., Turner G., The short range structure of sodium phosphate glasses I. MAS NMR studies, J. Non-Cryst. Solids, 1990, 116, 39-45.

[56] Walter G., Vogel J., Hoppe U., Hartmann, P., The structure of CaO$\mathrm{Na}_{2} \mathrm{O}-\mathrm{MgO}-\mathrm{P}_{2} \mathrm{O}_{5}$ invert glass, J. Non-Cryst. Solids, 2001, 296, 212-223.

[57] Hoppe U., A structural model for phosphate glasses, J. Non-Cryst. Solids, 1996, 195, 138-147.

[58] Hoppe U., Walter G., Kranold R., Stachel D., Structural specifics of phosphate glasses probed by diffraction methods: a review, J. Non-Cryst. Solids, 2000, 264, 29-47.

[59] Christie J.K., Brauer D., The role of fluoride in the nanoheterogeneity of bioactive glasses, Phys. Chem. Glasses, 2017, $58,180-186$

[60] Mead R.N., Mountjoy G., A Molecular Dynamics Study of the Atomic Structure of $(\mathrm{CaO})_{x}\left(\mathrm{SiO}_{2}\right)_{1-x}$ Glasses, The J. Phys. Chem. B, 2006, 110, 14273-14278.

[61] Christie J.K., Tilocca A., Integrating biological activity into radioisotope vectors: molecular dynamics models of yttrium-doped 
bioactive glasses, J. Mater. Chem., 2012, 22, 12023.

[62] Christie J.K., Tilocca A., Aluminosilicate glasses as yttrium vectors for in situ radiotherapy: Understanding compositiondurability effects through molecular dynamics simulations, Chem. Mater., 2010, 22, 3725-3734.
[63] Tilocca A., Structural models of bioactive glasses from molecular dynamics simulations, P. Roy. Soc. A- Math. Phys., 2009, 465, 1003-1027. 